\title{
Photocentric orbits from a direct combination of ground-based astrometry with Hipparcos
}

\section{Comparison with known orbits}

\author{
G. A. Gontcharov and O. V. Kiyaeva
}

Pulkovo observatory, Saint-Petersburg 196140, Russia

Received 13 December 2001 / Accepted 1 March 2002

\begin{abstract}
Complementing the Proper Motions of Fundamental Stars catalogue by Gontcharov et al. (CDS, I-266) the photocentric orbits of some FK5/Hipparcos stars are calculated in a direct combination of the Hipparcos data with astrometric ground-based observational catalogues having epochs later than 1939. Some capabilities and limitations of this method are discussed in a comparison of our results with known orbits of Sirius, Procyon, Rasalhague, $\mu$ Cas and others. We conclude that this direct combination is an acceptable tool to discover and investigate photocentric orbits with periods from 10 to 55 years and the semi-major axis of apparent ellipses $>0.08$ arcsec. It is particularly suitable for pairs with large magnitude difference as well as for calculation of the preliminary orbits of new astrometric binaries which will be considered in forthcoming papers. In this paper new photocentric orbits and component masses are calculated for 4 pairs: $\alpha \mathrm{UMa}, \beta \mathrm{LMi}, \delta$ And and $\xi$ Aqr.
\end{abstract}

Key words. stars: binaries: general - stars: fundamental parameters - star: individual: $\alpha \mathrm{UMa}$ - star: individual: $\beta$ LMi star: individual: $\delta$ And - star: individual: $\xi$ Aqr

\section{Introduction}

The double and multiple stellar systems with non-linear motion of the photocentre, detectable in series of ground-based astrometric observations over time intervals of some decades, are known as astrometric binaries. This motion is due to some fainter components of star-like masses and it is composed of the linear motion of the stellar system's barycentre and the elliptical motion of the photocentre around the barycentre. The classical examples of long-investigated astrometric binaries are the systems with white dwarfs, Sirius and Procyon, that are considered below. The variations of their proper motions were first investigated by Bessel (1844).

Astrometric binaries have been difficult to discover and investigate by classical astrometric observations because telescopes have different systematic errors that are unknown and variable with time. Therefore, the main problem was to establish a rather precise common reference frame for the many ground-based catalogues used. Only a few astrometric binaries were known before the Hipparcos mission (ESA 1997). The Hipparcos catalogue (hereafter HIP) gives us at least two ways to discover and investigate astrometric binaries:

- Hipparcos found thousands of stellar systems with nonlinear motion of their photocentre. Many of those systems are collected in parts $G$ (acceleration solution) and

Send offprint requests to: G. A. Gontcharov,

e-mail: georgegontcharov@yahoo.com
$\mathrm{X}$ (stochastic solution) of the Double and Multiple Systems Annex (DMSA) of the HIP. Part V (variability-induced movers) of the DMSA also contains some stellar systems with a non-linear motion of the photocentre, but which are generally non-elliptical, caused by the variable magnitude of a component. Some of the suspected non-single stars in the HIP ("S" in the field H61, solution quality) may also show non-linear motions of their photocentres;

- In order to discover and investigate astrometric binaries, ground-based catalogues can be compared and combined with the precise HIP by different methods. First, several hundred stars with considerable discrepancy between the HIP and ground-based proper motions were identified by many authors (e.g. Wielen 1997; Gontcharov \& Kornilov 1997) and might show non-linear motions of their photocentres. Second, the possible non-linear motion of a star can be taken into account statistically, as was done in the construction of the FK6 by Wielen et al. (1999). FK6 is a combination of the HIP with the best ground-based compilation, the FK5 (Fricke et al. 1988; Fricke et al. 1991). Third, many past observational ground-based catalogues can be combined directly with the HIP by forming series of positions of stars over decades of observations. Such series can reveal non-linearly moving stars.

The direct combination of the HIP with 57 observational ground-based catalogues having epochs between 1939 and 1995 has been realized for the 1535 stars of the Basic FK5 by 
Gontcharov et al. (2001). Preliminary results for five very interesting astrometric binaries have been presented by Gontcharov et al. (2000). The main task of the direct combination is a reduction of observational ground-based catalogues into a common reference frame close to the ICRS/Hipparcos. The parallaxes from the HIP, radial velocities from the Hipparcos input catalogue by Turon et al. (1993) as well as positions from the HIP and observational ground-based catalogues were used in the reduction. The proper motions from the HIP and several ground-based compilation catalogues were used as initial values for iterations. The results of these iterations are uniform series of star positions over several decades (including the photocentric positions from the HIP) and improved individual proper motions of the stars. These proper motions were published as The Proper Motions of Fundamental Stars catalogue (PMFS), Part I (Gontcharov et al. 2001) deposited as I-266 at the CDS.

A possible non-linear motion of several hundred photocentres was detected from the obtained series of positions. These stellar systems were marked in the PMFS as class "A" (astrometric binaries). The non-linear motion was separated into the linear motion of the barycentre of the stellar system (classical proper motion presented in the PMFS) and elliptical motion of the photocentre around the barycentre. The latter will be analyzed here and in subsequent papers with the aim to calculate new photocentric orbits and masses of components.

The procedure of separation of the non-linear motion into a linear and an elliptic one includes:

1. Elimination of the obtained proper motion from the series of positions and calculation of 6 orbital elements $(a, T, \Omega$, $\omega, e i$ ) by the method described by Kiyaeva \& Kalinichenko (1998). The orbital period was taken from external data or calculated from the series with a precision of 1-3 years. For some well-known pairs we used known values of some other elements marked in italics in the following tables;

2. Elimination of obtained orbital motion from the series of positions and calculation of the proper motion by the leastsquare method;

3. Steps 1-2 were repeated in iterations until the obtained improvements became insignificant.

The Basic FK5 contains several well-known orbital pairs with an appropriate period and semi-major axis of the apparent ellipse of the photocentric orbit. The comparison of our reduced astrometric observations and the derived orbital elements with the known orbits could help us to estimate capabilities and limitations of our observational material and method.

Most of the considered pairs are in the Sixth Catalog of Orbits of Visual Binary Stars by Hartkopf \& Mason (2001) (hereafter the Sixth catalogue). The speckle-interferometric observations of the pairs are taken from the Fourth Catalog of Interferometric Measurements of Binary Stars by Hartkopf et al. (2001) (hereafter the Fourth catalog), unless otherwise specified. However, several well-known orbital pairs have been eliminated from the observational lists of meridian telescopes and astrolabes a long time ago. For example, we did not get enough observations for the detection of the orbital motion of HIP 71681 ( $\alpha$ Cen A-B) and HIP 104214 (61 Cyg A-B). Some other well-known orbital pairs are members of multiple stellar systems with several components observable by meridian telescopes and astrolabes. These and some other more complicated cases will be considered in forthcoming papers.

\section{Comparison with known orbits}

Some known orbital pairs are compiled in Table 1. Table 2 gives the components $\mu_{\alpha \cdot \cos \delta}$ and $\mu_{\delta}$ of the proper motion of the considered stellar pairs taken from the PMFS, HIP and FK5 with the precision of 0.001 arcsec/year. The PMFS catalogue gives the barycentric proper motions (that of $\mu$ Cas has been corrected, see below). The FK5 gives the photocentric proper motions based on more than a century of observations, these motions are close to the barycentric ones for many stars. The proper motions of known pairs in the HIP are reduced to the photocentre, barycentre or components separately, depending on the results of the mission. In Table 2 the barycentric proper motion taken or calculated from the HIP is marked as $m$ whereas the photocentric one is marked as $p$. The Hipparcos proper motion of Procyon was recalculated by Girard et al. (2000) in a combination of Hipparcos and ground-based data.

The capabilities of our method can be inferred from Table 3 where we show the standard deviation of the residuals between the astrometric observations after their reduction to the ICRS and the orbits, and the "signal-noise ratio" $(S / N)$ between the semi-major axis of apparent ellipse $a_{\text {app }}$ and the standard deviation $\sigma_{\alpha+\delta}$ along the celestial great circle. The standard deviation $\sigma_{\alpha+\delta}$ together with those on the both coordinates, $\sigma_{\alpha}$ and $\sigma_{\delta}$, give an estimate of the accuracy of the catalogues used after their reduction to the ICRS (i.e. the accuracy of the formed series of the observations). Sirius and Procyon show rather large standard deviation probably because of their brightness. For the rest of the pairs the standard deviation is about of 0 . $^{\prime} 05$ for both $\alpha$ and $\delta$.

The precision of the calculated orbital elements $a, T, \Omega, \omega$, $e$ and $i$ is affected by 1 ) the orientation and other features of the orbit, 2) the accuracy of the formed series of the observations, 3 ) the number of catalogues in the formed series and 4) the distribution of the series along orbit. The latter appears rather important because the considered temporal interval of $\leq 56$ years usually includes only one or two periods and the distribution is uneven. To estimate the precision of the orbital elements we simulated the series of observations for the real observational epochs but randomly distributed around real series with a dispersion of 0 ' 05 for both $\alpha$ and $\delta$. The dispersion of the sets of the orbital elements derived from the simulated series is taken as the precision of the orbital elements. The number of the simulated series slightly affects the dispersion. We simulated 20 series for each pair.

The orbital elements were derived from our observational material for all the considered pairs. However, for the first 5 pairs in Table 3 some rather precise photocentric orbits have been derived from more extensive observational material (mainly photographic observations with large refractors). Therefore, as expected, the precision of our orbit for these pairs is less than that of a known orbit, and these orbits practically coincide in the apparent plane. Thus, for these 5 pairs we only compare our observational material with the known orbits and 
Table 1. Stellar systems under consideration: numbers from various catalogues, spectrum of primary, visual magnitude of primary, parallax from HIP, accepted parallax and orbit grade taken from the Sixth catalogue: from definitive (1) to indeterminate (5), 9 is for photocentric orbit.

\begin{tabular}{llrrrrrlrlcc}
\hline \hline Name & Other name & HIP & FK5 & HD & GL & ADS & Sp of A & $V_{\mathrm{A}}^{\mathrm{m}}$ & $\pi_{\mathrm{HIP}}$ & $\pi$ accepted & Orbit grade \\
\hline$\alpha \mathrm{CMa}$ & Sirius & 32349 & 257 & 48915 & $244 \mathrm{~A}$ & 5423 & A0V & -1.4 & 0.379 & 0.379 & 3 \\
$\alpha \mathrm{CMi}$ & Procyon & 37279 & 291 & 61421 & $280 \mathrm{~A}$ & 6251 & F5IV-V & 0.4 & 0.286 & 0.283 & 3 \\
$\mu \mathrm{Cas}$ & & 5336 & 1030 & 6582 & $53 \mathrm{~A}$ & & G5VI & 5.2 & 0.132 & 0.132 & 5 \\
$\alpha$ Oph & Rasalhague & 86032 & 656 & 159561 & 681 & & A5IV & 2.1 & 0.070 & 0.070 & 9 \\
$10 \mathrm{UMa}$ & & 44248 & 339 & 76943 & $332 \mathrm{~A}$ & & F5V & 4.1 & 0.061 & 0.061 & 1 \\
$\delta \mathrm{And}$ & & 3092 & 20 & 3627 & & 548 & K3III & 3.3 & 0.032 & 0.032 & \\
$\alpha \mathrm{UMa}$ & Dubhe & 54061 & 417 & 95689 & & 8035 & K0III & 2.0 & 0.026 & 0.026 & 3 \\
$\beta \mathrm{LMi}$ & & 51233 & 390 & 90537 & & 7780 & G8III-IV & 4.6 & 0.022 & 0.022 & 2 \\
$\xi \mathrm{Aqr}$ & & 106786 & 1569 & 205767 & & & A7V & 4.7 & 0.018 & 0.022 & \\
\hline
\end{tabular}

Table 2. The proper motion of the systems (in arcsec): from the PMFS (barycentric), HIP ( $m$ - barycentric, $p$ - photocentric) and FK5 (longterm photocentric).

\begin{tabular}{lcccc}
\hline \hline Name & PMFS & HIP & FK5 \\
\hline & $\mu_{\alpha \cdot \cos \delta} \mu_{\delta}$ & $\mu_{\alpha \cdot \cos \delta} \mu_{\delta}$ & $\mu_{\alpha \cdot \cos \delta} \mu_{\delta}$ \\
\hline$\alpha \mathrm{CMa}$ & $-0.551-1.218$ & $-0.546-1.223 m$ & $-0.553-1.205$ \\
$\alpha \mathrm{CMi}$ & $-0.704-1.028$ & $-0.711-1.021 m$ & $-0.710-1.023$ \\
$\mu \mathrm{Cas}$ & $+3.425-1.597$ & $+3.421-1.599 m$ & $+3.424-1.596$ \\
$\alpha \mathrm{Oph}$ & $+0.120-0.232$ & $+0.110-0.223 p$ & $+0.120-0.226$ \\
$10 \mathrm{UMa}$ & $-0.435-0.239$ & $-0.435-0.245 m$ & $-0.440-0.246$ \\
$\delta \mathrm{And}$ & $+0.138-0.088$ & $+0.115-0.083 p$ & $+0.136-0.091$ \\
$\alpha \mathrm{UMa}$ & $-0.115-0.060$ & $-0.126-0.068 m$ & $-0.119-0.066$ \\
$\beta \mathrm{LMi}$ & $-0.121-0.104$ & $-0.119-0.104 m$ & $-0.118-0.101$ \\
$\xi \mathrm{Aqr}$ & $+0.114-0.021$ & $+0.114-0.024 p$ & $+0.116-0.025$ \\
\hline
\end{tabular}

Table 3. Standard deviation (in arcsec) of the astrometric observations from orbits, and the "signal-noise ratio" $(S / N)$ of the semi-major axis of apparent ellipse $a_{\text {app }}$ to the standard deviation $\sigma_{\alpha+\delta}$ along celestial great circle.

\begin{tabular}{lccccrl}
\hline \hline Name & $a_{\text {app }}$ & $\sigma_{\alpha+\delta}$ & $\sigma_{\alpha}$ & $\sigma_{\delta}$ & $S / N$ & Orbit from \\
\hline$\alpha \mathrm{CMa}$ & 2.400 & 0.201 & 0.084 & 0.183 & 11.9 & Benest \& Duvent (1995) \\
$\alpha \mathrm{CMi}$ & 1.100 & 0.161 & 0.096 & 0.130 & 6.8 & Girard et al. (2000) \\
$\mu \mathrm{Cas}$ & 0.190 & 0.076 & 0.057 & 0.048 & 2.5 & Drumond et al. (1995) \\
$\alpha \mathrm{Oph}$ & 0.070 & 0.050 & 0.034 & 0.037 & 1.4 & Augensen \& Heintz (1992) \\
$10 \mathrm{UMa}$ & 0.175 & 0.070 & 0.046 & 0.059 & 2.5 & Heintz (1997) \\
$\delta \mathrm{And}$ & 0.280 & 0.071 & 0.062 & 0.042 & 3.9 & this paper \\
$\alpha \mathrm{UMa}$ & 0.200 & 0.044 & 0.032 & 0.029 & 4.5 & this paper \\
$\beta \mathrm{LMi}$ & 0.110 & 0.058 & 0.050 & 0.035 & 1.9 & this paper \\
$\xi \mathrm{Aqr}$ & 0.080 & 0.053 & 0.029 & 0.045 & 1.5 & this paper \\
\hline
\end{tabular}

disregard some unreliable orbits of Procyon and $\mu$ Cas because of their large deviation from our observational material.

For $\delta$ And, $\alpha$ UMa, $\beta$ LMi and $\xi$ Aqr our observational material has allowed us to derive new photocentric orbits and component masses. For these pairs the self-consistent sets of orbital elements and other parameters are presented pair-bypair in Tables 6, 7, 9 and 10. To obtain the sets we used the following common relations. The elements $P, T, i, e$ and $\Omega$ are the same for the relative (fainter (B) with respect to brighter (A) component) and photocentric (photocentre with respect to barycentre) orbits; $\omega$ differs by $180^{\circ}$. The ratio of the relative $\left(a_{\mathrm{BA}}\right)$ and photocentric $\left(a_{\mathrm{pm}}\right)$ semi-major axes (both in arcsec hereafter) depends on the mass ratio and the magnitude difference of the components. Both components, barycentre and photocentre, are on a line; the ratio of the distance "barycentreA" to " $\mathrm{B}-\mathrm{A}$ " is $B=M_{\mathrm{B}} /\left(M_{\mathrm{A}}+M_{\mathrm{B}}\right)$, where $M_{\mathrm{A}}$ and $M_{\mathrm{B}}$ are the component masses; the ratio of the distance "photocentre-A" to " $\mathrm{B}-\mathrm{A}$ " is $\beta=1 /\left(1+10^{0.4 \Delta m}\right)$, where $\Delta \mathrm{m}$ is the magnitude difference. Thus, $a_{\mathrm{pm}}=a_{\mathrm{BA}} \cdot(B-\beta)$. For every pair the system of 2 equations

$M_{\mathrm{A}}+M_{\mathrm{B}}=a_{\mathrm{BA}}^{3} /\left(\pi^{3} P^{2}\right),(\pi=$ parallax, $P=$ period $)$,
$a_{\mathrm{pm}}=a_{\mathrm{BA}} \cdot\left(M_{\mathrm{B}} /\left(M_{\mathrm{A}}+M_{\mathrm{B}}\right)-1 /\left(1+10^{0.4 \Delta m}\right)\right)$

was solved 1) accurately for the component masses $M_{\mathrm{A}}$ and $M_{\mathrm{B}}$ if the fainter component has been observed and $a_{\mathrm{BA}}$ is known or 2) less accurately for the mass of a component and $a_{\mathrm{BA}}$ if the other mass is approximately estimated from the magnitude, spectrum, color index or other external data using the tables by Allen (1973). The uncertainties of accepted values are 


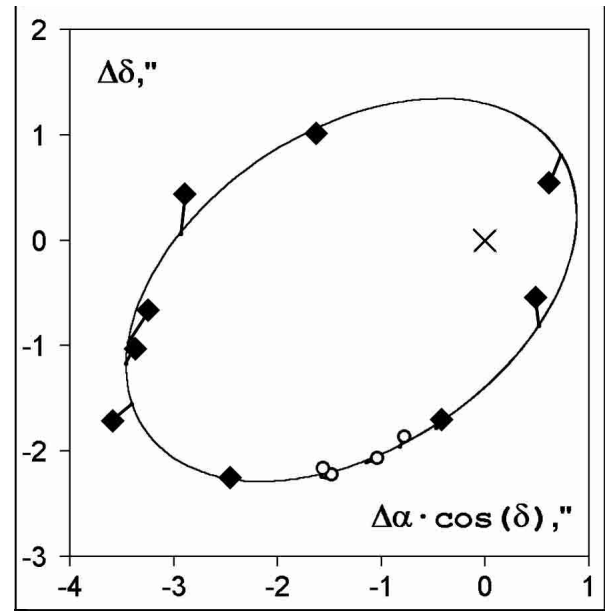

Fig. 1. The photocentric orbit of Sirius by Benest \& Duvent (1995) and our reduced astrometric observations. The observations from the Carlsberg meridian catalogues are shown by open circles.

taken into account in estimating the precision of the derived parameters provided in the following tables pair-by-pair. All the masses in the tables are given in solar masses.

Our observational material together with the derived and some known photocentric orbits are shown in the following figures which are composed of three subfigures. The first subfigure (the only one for Sirius and Procyon) shows the observations together with the derived and known apparent ellipses in the form of the $\Delta \alpha \cos \delta-\Delta \delta$-differences between the photocentres and the barycentres (i.e. apparent ellipses of the photocentric orbit). The observations are connected by straight lines to the appropriate epochs on the derived or best known orbital ellipses. The second and third subfigures give the differences $\Delta \alpha \cos \delta$ and $\Delta \delta$, respectively, as a function of time. $\Delta \alpha \cos \delta$ and $\Delta \delta$ are given in arcsec.

\section{1. $\alpha$ CMa (Sirius)}

Sirius is a well-known pair of a main sequence star and a white dwarf. The photocentre coincides with the main sequence star. Orbits by Volet (1932), van den Bos (1960), Gatewood \& Gatewood (1978) and Benest \& Duvent (1995) are very similar. The latter (with photocentric semi-major axis of 2".49) is shown in Fig. 1 together with our reduced astrometric observations. The observations were rare because the star was usually eliminated from observational lists because of its duplicity and brightness.

Bonnet-Bidaud et al. (2000) argue that no third component exists in this system. Observations from the Carlsberg meridian catalogues 1-4 (open circles in Fig. 1) also perfectly fall into the known orbits providing therefore no indication of a third component.

\section{2. $\alpha \mathrm{CMi}$ (Procyon)}

Procyon is a well-known pair consisting of an F5IV-V star and a white dwarf. Photocentric orbits by Strand (1951), Irwin et al. (1992) and Girard et al. (2000) are shown in Fig. 2 as dashed,
Table 4. Orbital elements and parameters of Procyon.

\begin{tabular}{|c|c|c|c|}
\hline & 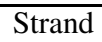 & Irwin et al. & Girard et al. \\
\hline$a_{\mathrm{pm}}$ & $1 . .217$ & 1". 179 & $1^{\prime \prime} .232$ \\
\hline$P$ & 40.65 & 40.38 & 40.82 \\
\hline$T$ & 1968.3 & 1967.86 & 1967.97 \\
\hline$\Omega$ & $104^{\circ} .3$ & 104.8 & 97.3 \\
\hline$\omega$ & 89.8 & 88.8 & 92.2 \\
\hline$e$ & 0.40 & 0.365 & 0.407 \\
\hline$i$ & $35^{\circ} .7$ & 31.9 & 31.1 \\
\hline$a_{\mathrm{BA}}$ & 4. 548 & 4.. 496 & 4.' 296 \\
\hline parallax & 0.287 & 0.286 & 0.283 \\
\hline$\sum M$ & 2.37 & 2.37 & 2.10 \\
\hline mass of $\mathrm{A}$ & 1.74 & 1.75 & 1.50 \\
\hline mass of $B$ & 0.63 & 0.62 & 0.60 \\
\hline$\sigma_{\alpha+\delta}$ & 0.'204 & 0.209 & 0.161 \\
\hline
\end{tabular}

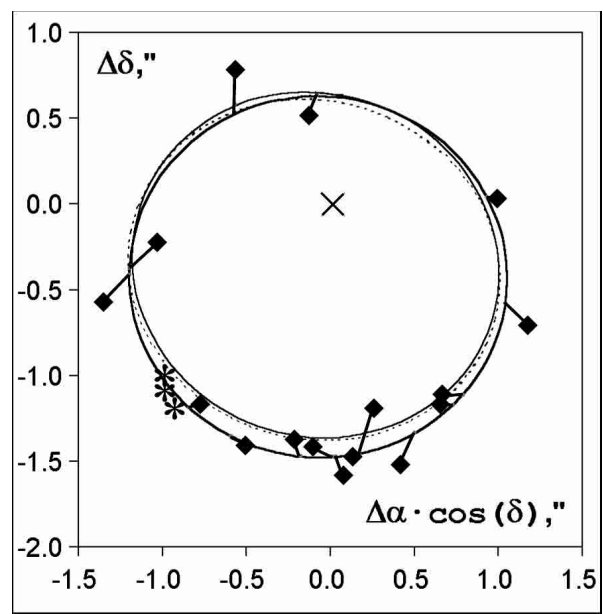

Fig. 2. Three photocentric orbits of Procyon, our observational material (diamonds) and 3 relative observations with the assumption $B-\beta=0.287$ (asterisks).

thin and thick ellipses, respectively. These orbits are rather different. The orbit by Girard et al. should be the most precise one since it is based on photographic astrometric observations spanning 83 years, Hipparcos observations of the photocentre as well as 2 observations of component B relative to A by HST and a coronagraph. The latter observations together with a coronagraphic observation by Walker et al. (1994) are shown in Fig. 2 as asterisks with the assumption $B-\beta=0.287$ obtained from the masses provided by Girard et al. Our observational material is shown in Fig. 2 as filled diamonds.

Orbital elements and other parameters of the orbits are shown in Table 4 together with the standard deviation $\sigma_{\alpha+\delta}$ of the observations from the orbits along celestial great circles. The orbit by Girard et al. much better fits our results.

\section{3. $\mu$ Cas}

This is a nearby high velocity population II star and is thus interesting because of the importance of determining the masses, luminosities, and metal abundances of its components. It is the only pair for which we had to recalculate the barycentric proper motion after the publication of the PMFS catalogue due 
Table 5. Orbital elements and parameters of $\mu$ Cas.

\begin{tabular}{|c|c|c|}
\hline & McCarthy et al. & Drummond et al. \\
\hline$\overline{a \mathrm{pm}}$ & 0.20 & 0.191 \\
\hline$P$ & 23.5 & 21.75 \\
\hline$T$ & 1977.6 & 1975.74 \\
\hline$\Omega$ & $54^{\circ} .3$ & 47.3 \\
\hline$\omega$ & $186^{\circ} .1$ & 152.7 \\
\hline$e$ & 0.46 & 0.56 \\
\hline$i$ & 104.8 & $110^{\circ} 0$ \\
\hline$a_{\mathrm{BA}}$ & 0.97 & 1..01 \\
\hline parallax & 0.137 & 0.133 \\
\hline$\sum M$ & 0.73 & 0.92 \\
\hline mass of $A$ & 0.58 & 0.74 \\
\hline mass of $B$ & 0.15 & 0.17 \\
\hline$\sigma_{\alpha+\delta}$ & 0.100 & 0.076 \\
\hline
\end{tabular}

to some new data. Now we use $\mu_{\alpha \cdot \cos \delta}=+3.420 \mathrm{arcsec} /$ year instead of +3.425 in the PMFS and $\mu_{\delta}=-1.593 \mathrm{arcsec} / \mathrm{year}$ instead of -1.597 .

Speckle-interferometric observations we have dating since 1973 as well as spectroscopic ones as mentioned by Drummond et al. (1995). However, according to the Fourth catalog this pair has never been resolved at periastron (in 1975 and 1997). There are two series of astrometric observations of the photocentre (component A): at the Sproul observatory by Lippincott (1981) and more recently by Heintz \& Cantor (1994), and at the Allegheny observatory by Russell \& Gatewood (1984). These series, either one or both, were the basis for many orbit determinations, such as those by Lippincott (1981), Russell \& Gatewood (1984), McCarthy et al. (1993), Heintz \& Cantor (1994), Drummond et al. (1995) or Gudehus (2001). All orbits, except that of McCarthy et al., are rather similar. The standard deviation of our reduced astrometric observations from the "similar" orbits is about 0 '. 08 , and from the orbit by McCarthy et al. it is 0 .' 10 . The orbits by McCarthy et al. and Drummond et al. are presented in Table 5 as well as in Fig. 3 by dashed and solid curves, respectively. All other apparent ellipses practically coincide with the one by Drummond et al. Our observational material is shown by diamonds. All successful speckle-interferometric observations with the assumption $B-\beta=0.189$ are shown by asterisks.

All our reduced astrometric observations with epochs near periastron passage in 1975 show some systematic deviation from the known orbits, although within a priori estimated accuracy of the ground-based catalogues. It may argue for a slightly different $\Omega$ or an additional long-period non-linear motion of the photocentre hardly distinguishable from the perspective acceleration $\mathrm{d} \mu / \mathrm{d} t$ of this fast nearby star. Since our data are not numerous enough to recalculate this acceleration, we have used the standard values. Lippincott (1981) argues for a value of this acceleration differing from the standard value. It may be explained by a third disturbing body of the system.

\section{4. $\alpha$ Oph (Rasalhague)}

Two rather different photocentric orbits of this pair were derived by Kamper et al. (1989) and Augensen \& Heintz (1992), respectively, based on photographic observations of the photocentre and some spectroscopic ones. A few interferometric observations have been analyzed by Kamper et al. and by Boccaletti et al. (2001). Our reduced astrometric observations give the same standard deviations from both orbits. The observations together with the orbit by Augensen and Heintz are shown in Fig. 4. The Hipparcos results are presented by 3 open diamonds perfectly falling onto the orbit.

The orbital period is about 8.6 years. Most astrometric catalogues (except Hipparcos and Carlsberg meridian catalogues) give an average of observations unevenly distributed over several years. Therefore, the catalogues give results corresponding not to a point but rather a long part of the orbit. The corresponding error appears in the case of Rasalhague and limits our method: apparently no useful results can be obtained for binaries with shorter periods.

\section{5. $10 \mathrm{UMa}$}

A lot of interferometric observations since 1975 cover well the orbit of this pair in the Lynx constellation. Moreover, the components of $4.0^{\mathrm{m}}$ and $6.3^{\mathrm{m}}$ have been observed separately with a large number of telescopes used for astrometric and astrophysical observations. As a result, this is the best orbit among the FK5 stars determined by visual observations, and the photocentric semi-major axis is also known. The authors of the FK5 (where this star is named as Bradley 1268 Lyncis) recommended to take into account the orbit by Baize (1955) in any calculations. Some observers followed this recommendation as seen from the results and descriptions of groundbased catalogues. However, in most such cases, for example in the Carlsberg meridian catalogues (1997), the observed coordinates may depend on the FK5 coordinates (and, consequently, the assumed orbit) as well as on the contribution of the fainter component, depending on many factors. Therefore, it is difficult to conclude to which point of the pair the observations actually refer. It is pointed out in the Carlsberg catalogues that the component A was observed. It must be a mistake because the component B was always inside the instrument slit together with the component A. Analyzing the Carlsberg results we have to assume that the positions in the CMC2 and CMC4 catalogues hold for the barycentre and those in the CMC3 and CMC5 catalogues for the component A. Then we calculated the positions of the photocentre using magnitude differences and component masses given by Soderhjelm (1999). Of course, some uncertainty and sources of mistakes remain. Therefore, our results for this pair should be considered with caution.

The best 3 orbits by Martin et al. (1998), Heintz (1997) and Soderhjelm (1999) practically coincide in the apparent plane. All 3 orbits are shown as one ellipse with a photocentric semimajor axis of 0 .'18 in Fig. 5 together with our reduced astrometric observations (filled diamonds) and a linear motion of the photocentre calculated from the HIP (3 open diamonds). The photocentric semi-major axis remains slightly uncertain: Martin et al. and Heintz give 0.'175 whereas Soderhjelm gives 0.214. Breakiron et al. (1975) also obtained a photocentric semi-major axis of 0.20 with the Allegheny refractor. 

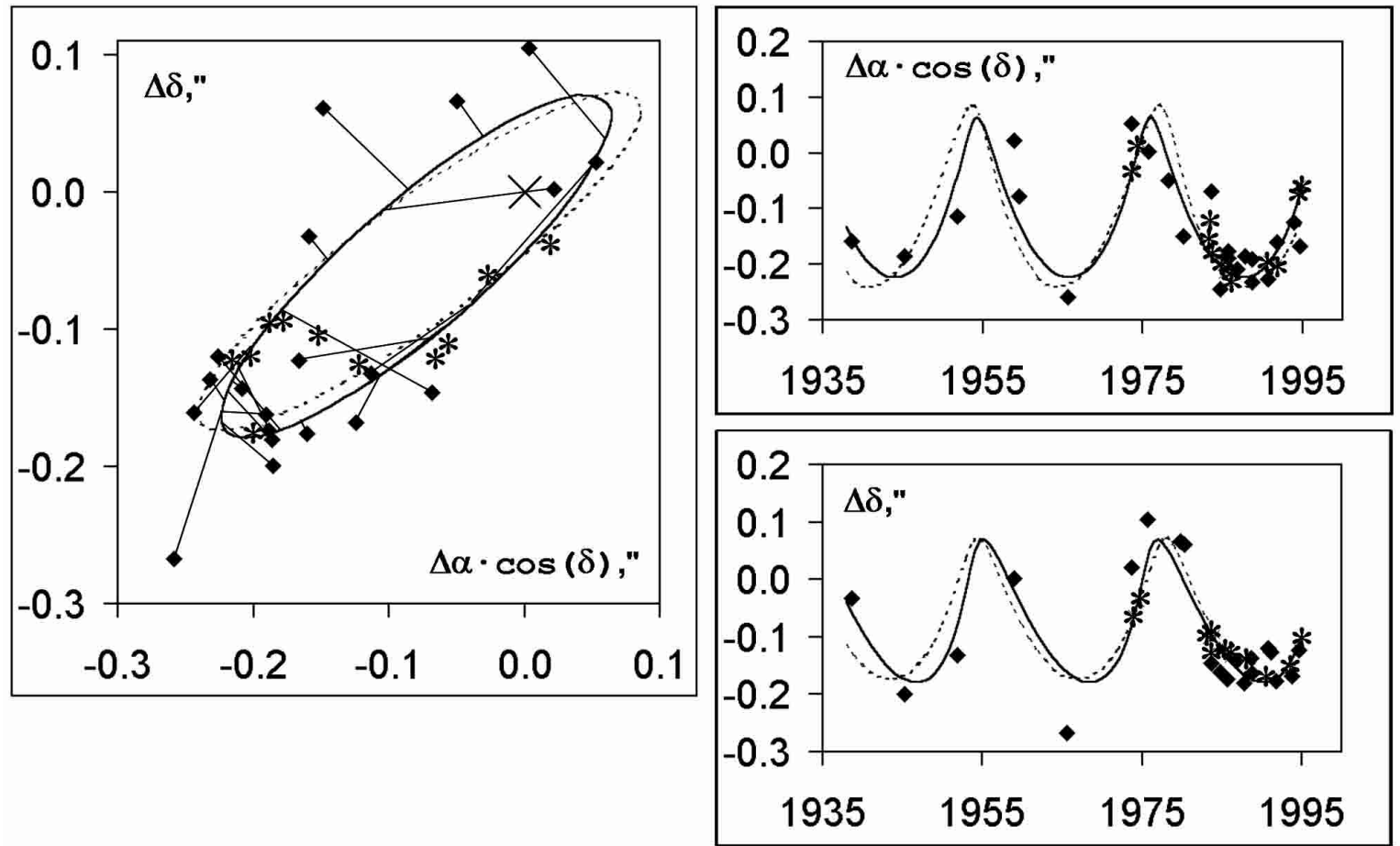

Fig. 3. The photocentric orbits of $\mu$ Cas by McCarthy et al. (1993) (dashed curve) and Drummond et al. (1995) (solid curve) as well as our observational material (diamonds) and relative observations with the assumption $B-\beta=0.189$ (asterisks).
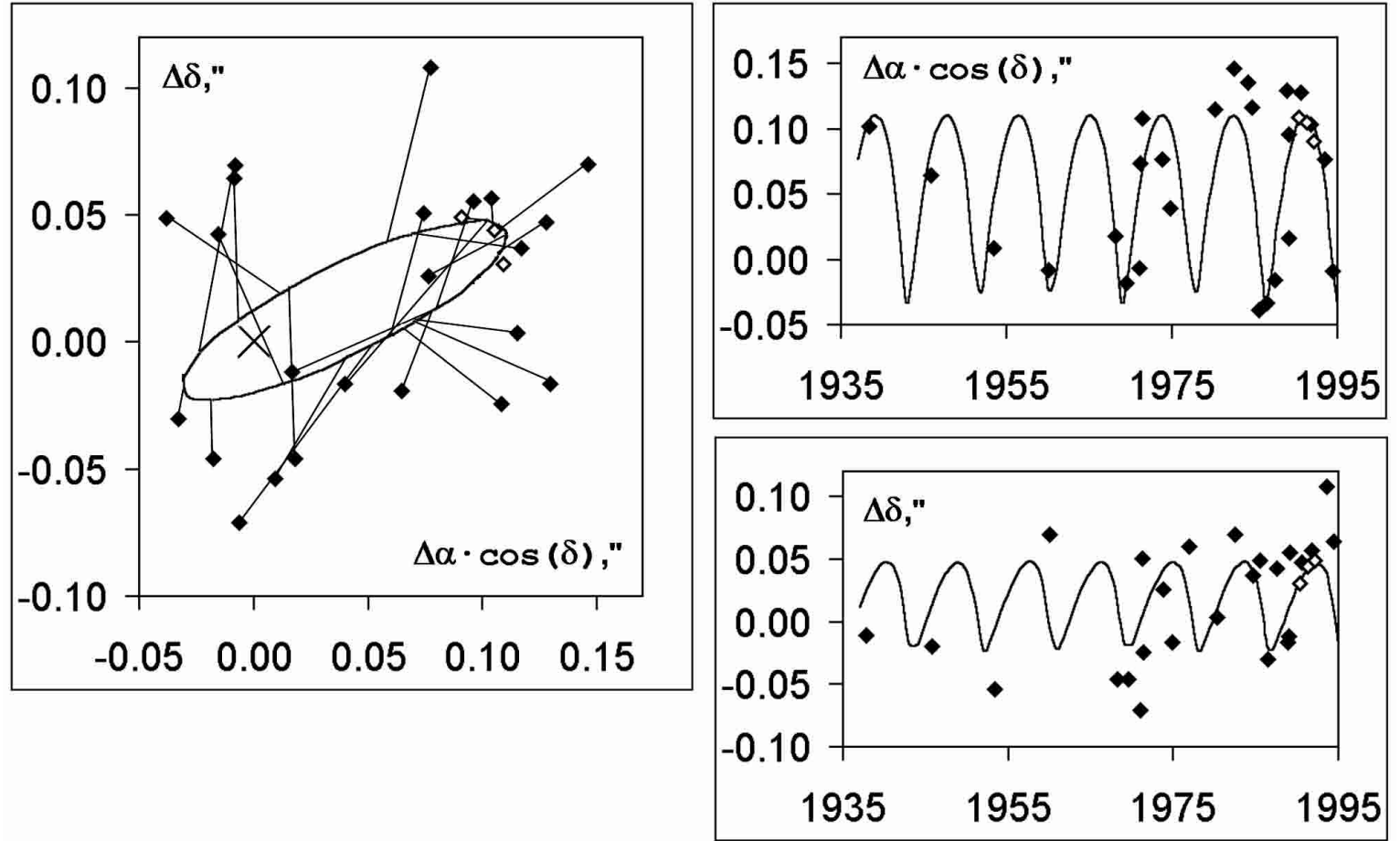

Fig. 4. The photocentric orbit of Rasalhague by Augensen \& Heintz (1992) together with our ground-based (filled diamonds) and Hipparcos (3 open diamonds) astrometric results.

\section{6. $\delta$ And}

This is a triple or quadruple system with several interesting features: strong high-velocity wind, infrared excess, dust shell approximately between the secondary at several AU and the tertiary at a thousand AU according to Judge et al. (1987).
A dynamical interaction between the companions and the shell may be expected. $[\mathrm{Fe} / \mathrm{H}]=+0.16$ according to Taylor (1999).

Only the secondary can cause the elliptical motion of the photocentre which coincides with the primary. Judge et al. argue for the secondary being a white dwarf. It has, however, never been observed, even in many speckle-interferometric 

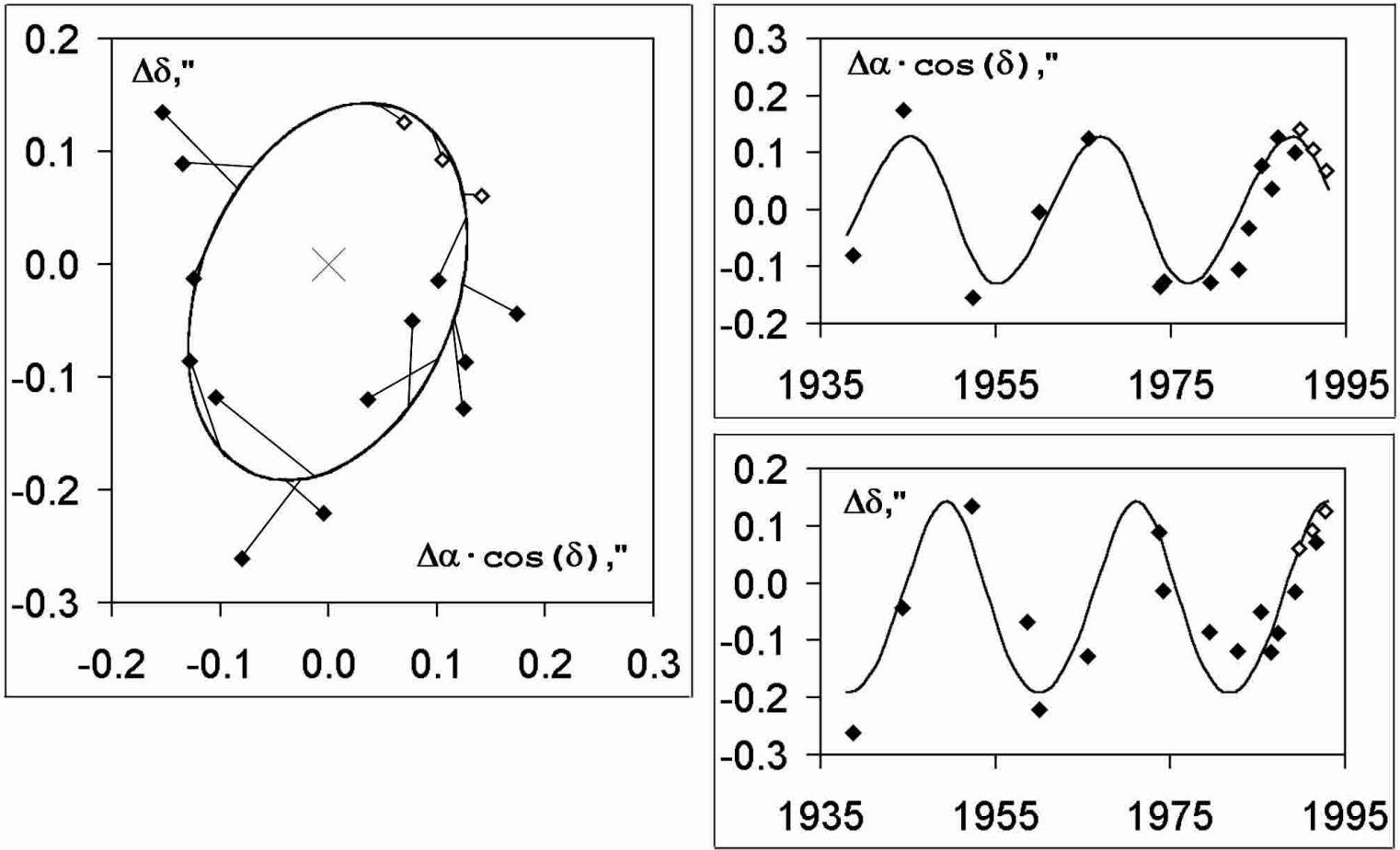

Fig. 5. A photocentric orbit of 10 UMa with semi-major axis of 0 ' 18 together with our ground-based (filled diamonds) and Hipparcos ( 3 open diamonds) astrometric results.

observations since 1976 as pointed out in the Fourth catalog. The HIP gives a linear motion of the photocentre. Therefore, the epoch 1991.25 should be far from a periastron passage moment.

The primary-secondary pair is known as a spectroscopic binary with single lines. It has been concluded from spectroscopic observations by R. F. Griffin (2001) that the semiamplitude of the radial velocity variations is near $4 \mathrm{~km} \mathrm{~s}^{-1}$ and the period is near 53 years (instead of 55.19 years as mentioned by Tokovinin 1997). Assuming $e<0.6$ and $i$ far from $90^{\circ}$ one finds a rather large photocentric semi-major axis, namely $6-$ 12 AU, or 0.'2-0!'4 with the parallax 0.'032 from the HIP. This is in perfect agreement with the photocentric semi-major axis 0 '.32 obtained by us. Thus, both astrometric and spectroscopic results of $a_{\mathrm{pm}}$ involve a rather large mass of the unseen secondary and a rather low mass of the primary. In order to get an acceptable mass of the giant primary one has to assume a mass of the secondary close to the white dwarf mass limit. Assuming 1.3 solar masses for the secondary we obtained the same mass of the primary and 0.' 62 for the semi-major axis of the relative orbit. The uncertainty of these two determined parameters is mainly due to the uncertainty of the photocentric semi-major axis.

Our astrometric orbit is shown in Fig. 6. The sets of parameters of the pair as derived from our results and as given in the MSC by Tokovinin (1997) are presented in Table 6. The latter set is based on the spectroscopic orbit and an assumption for the primary mass. This set should be disregarded. Our results give the first estimation of the photocentric semi-major axis and masses of the components from astrometric observations. Most important is the perfect agreement between the spectroscopic
Table 6. Two sets of parameters for $\delta$ And.

\begin{tabular}{lll}
\hline \hline & MSC & our \\
\hline$a_{\mathrm{pm}}$ & $>00^{\prime} 03$ & $0^{\prime} \cdot 32 \pm 00^{\prime} 03$ \\
$P$ & 55.19 & 52.8 \\
$T$ & 1901.5 & $1958.5 \pm 1.1$ \\
$\Omega$ & & $290^{\circ} \pm 7^{\circ}$ \\
$\omega$ & $356^{\circ} .1$ & $231^{\circ} \pm 9^{\circ}$ \\
$e$ & 0.34 & $0.5 \pm 0.1$ \\
$i$ & & $137^{\circ} \pm 5^{\circ}$ \\
$a_{\mathrm{BA}}$ & $0^{\prime} \cdot 83$ & $0^{\prime} \cdot 62 \pm 00^{\prime} 04$ \\
parallax & $0^{\prime} 034$ & $0^{\prime} 032 \pm 00^{\prime} 001$ \\
$\sum M$ & $>4.8$ & $2.6 \pm 0.4$ \\
mass of A & 4.6 & $1.3 \pm 0.4$ \\
mass of B & $>0.2$ & 1.3 \\
$B$ & $>0.04$ & $0.5 \pm 0.1$ \\
$\beta$ & 0 & 0 \\
Spectrum of A & K3III & K3III \\
Spectrum of B & & white dwarf \\
$M_{V}$ of A & $1.0^{\mathrm{m}}$ & $0.9^{\mathrm{m}}$ \\
$M_{V}$ of B & & $>5^{\mathrm{m}}$ \\
\hline
\end{tabular}

and astrometric estimates of the photocentric semi-major axis and, consequently, masses, although the orbital elements $T$ and $e$ are also similar.

\section{7. $\alpha$ UMa (Dubhe)}

This is a well-known visual and spectroscopic pair with several speckle-interferometric observations in the Fourth catalog. There are 2 orbits: one by Heintz (1963) supplemented by Tokovinin (1997) (hereafter "H-T") and another one by 

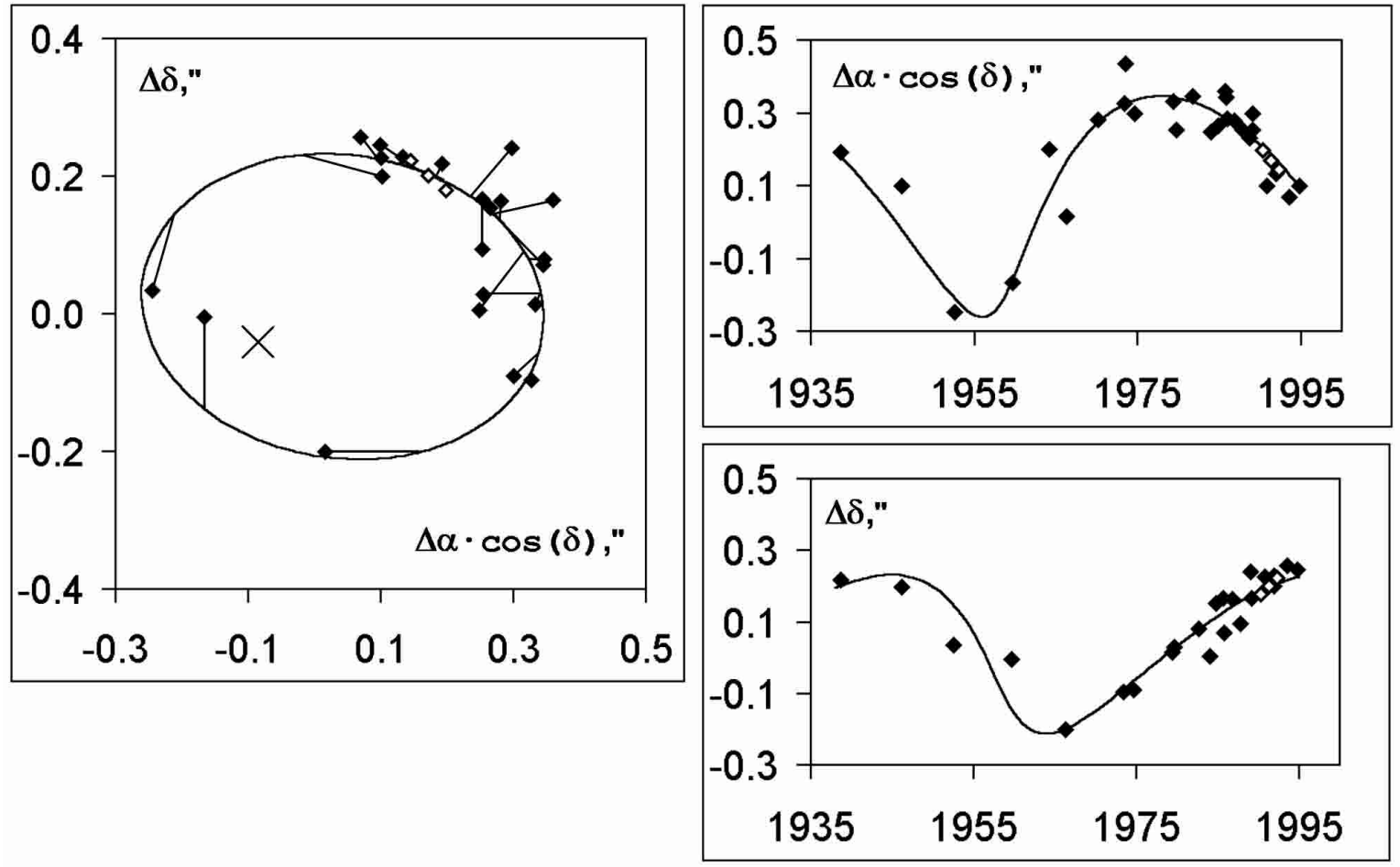

Fig. 6. Our photocentric orbit of $\delta$ And together with ground-based (filled diamonds) and Hipparcos ( 3 open diamonds) astrometric results.

Soderhjelm (1999) as mentioned in the Sixth catalog. However, the photocentric semi-major axis and, consequently, the masses of the components were never determined from observations before our research ( $\mathrm{H}-\mathrm{T}$ assume $M_{\mathrm{A}}$ ). Moreover, the orbital inclination is low and there are only a few observations near periastron. As a result, $\Omega, \omega$ and $i$ were poorly known: $\mathrm{H}-\mathrm{T}$ assume $\omega=180^{\circ}$, Soderhjelm assumes $\Omega=0^{\circ}, i=180^{\circ}$. A periastron passage is expected in 2002 .

Three photocentric orbits are presented in Table 7 and Fig. $7\left(a_{\mathrm{pm}}=0\right.$.'21 for all orbits in the figure): H-T (dashed curves), Soderhjelm (1999) (thin curves) and our orbit (thick curves). With the observations fixed, the barycentres are shown by a cross, a bold point and an oblique cross, correspondingly. The results of the relative observations with assumed $B-\beta=0.34$ are shown by asterisks. They are in good agreement with our reduced astrometric observations and orbit. The orbits are compared with these observations in Table 8 . The orbit by H-T should be denied because of the large deviation, although in fact it is due to a different $T$ only.

The uncertainty of the obtained component masses is mainly due to the uncertainty of the parallax. The obtained mass of the component $\mathrm{A}$ is in good agreement with its spectrum K0III. However, there is a disagreement between the known spectrum and absolute magnitude of the component $\mathrm{A}$. Perhaps it relates to $[\mathrm{Fe} / \mathrm{H}]=-0.13$ according to Taylor (1999). The spectrum of the component B can be estimated from its absolute magnitude and the obtained mass as about A3V. It is not so far from A8V which was estimated by Kondo et al. (1977) from the joint spectrum of the pair.
Table 7. Three sets of parameters of Dubhe.

\begin{tabular}{|c|c|c|c|}
\hline & $\mathrm{H}-\mathrm{T}$ & Soderhjelm & our \\
\hline$\overline{a_{\mathrm{pm}}}$ & $0 ! 14$ & & $0 !^{\prime} 21 \pm 0.02$ \\
\hline$P$ & 44.4 & 44.5 & 44.5 \\
\hline$T$ & 1956.8 & 1958 & 1958 \\
\hline$\Omega$ & $320^{\circ} 3$ & $0^{\circ}$ & $318^{\circ} \pm 24^{\circ}$ \\
\hline$\omega$ & $180^{\circ}$ & $222^{\circ}$ & $174^{\circ} \pm 23^{\circ}$ \\
\hline$e$ & 0.4 & 0.39 & 0.4 \\
\hline$i$ & $152^{\circ}$ & $180^{\circ}$ & $158^{\circ} \pm 14^{\circ}$ \\
\hline$a_{\mathrm{AB}}$ & 0.60 & 0.59 & 0.60 \\
\hline parallax & 0.027 & 0.026 & $0.026 \pm 0.001$ \\
\hline$\sum M$ & 5.7 & 5.94 & 6.2 \\
\hline mass of $\mathrm{A}$ & 4.0 & & $3.7 \pm 0.4$ \\
\hline mass of B & 1.7 & & $2.5 \pm 0.4$ \\
\hline$B$ & 0.30 & & $0.40 \pm 0.04$ \\
\hline$\Delta m$ & $2.94^{\mathrm{m}}$ & $2.98^{\mathrm{m}}$ & $3^{\mathrm{m}}$ \\
\hline$\beta$ & 0.063 & 0.060 & 0.06 \\
\hline Spectrum of A & K0III & & K0III \\
\hline Spectrum of B & FOV & & about $\mathrm{A} 3 \mathrm{~V}$ \\
\hline$M_{V}$ of $\mathrm{A}$ & $-0.9^{\mathrm{m}}$ & & $-0.9^{\mathrm{m}}$ \\
\hline$M_{V}$ of B & $2.0^{\mathrm{m}}$ & & $2.1^{\mathrm{m}}$ \\
\hline
\end{tabular}

Table 8. Standard deviation of our reduced astrometric observations from orbits.

\begin{tabular}{|c|c|c|c|}
\hline & $\sigma_{\alpha}$ & $\sigma_{\delta}$ & $\sigma_{\alpha+\delta}$ \\
\hline $\mathrm{H}-\mathrm{T}\left(a_{\mathrm{pm}}=0{ }^{\prime}{ }^{\prime} 14\right)$ & 0.081 & 0.053 & 0.096 \\
\hline $\mathrm{H}-\mathrm{T}\left(a_{\mathrm{pm}}=0^{\prime} 21\right)$ & 0.039 & 0.036 & 0.054 \\
\hline Soderhjelm $\left(a_{\mathrm{pm}}=0.21\right)$ & 0.033 & 0.032 & 0.046 \\
\hline our orbit & 0.'032 & 0.029 & $0{ }^{\prime} 044$ \\
\hline
\end{tabular}


Table 9. Three sets of parameters of $\beta$ LMi.

\begin{tabular}{|c|c|c|c|}
\hline & Soderhjelm & 6th catalog & our \\
\hline$a_{\mathrm{pm}}$ & & & $0.13 \pm 0^{\prime}{ }^{\prime} 04$ \\
\hline$P$ & 39 & 38.62 & 38.35 \\
\hline$T$ & 1998 & 1999.15 & 1999.33 \\
\hline$\Omega$ & $42^{\circ}$ & $41.5^{\circ}$ & $40^{\circ} \pm 15^{\circ}$ \\
\hline$\omega$ & $24^{\circ}$ & $29.8^{\circ}$ & $41^{\circ} \pm 12^{\circ}$ \\
\hline$e$ & 0.69 & 0.668 & $0.7 \pm 0.3$ \\
\hline$i$ & $79^{\circ}$ & $79.1^{\circ}$ & $81^{\circ} \pm 11^{\circ}$ \\
\hline$a_{\mathrm{AB}}$ & 0.3 & 0.363 & 0.36 \\
\hline parallax & o.'022 & $0 .{ }^{\prime} 022$ & $0.022 \pm 0.001$ \\
\hline$\sum M$ & 2.63 & 3.01 & 3.0 \\
\hline mass of $\mathrm{A}$ & & & $1.3 \pm 0.4$ \\
\hline mass of $B$ & & & $1.7 \pm 0.4$ \\
\hline$B$ & & & $0.57 \pm 0.07$ \\
\hline$\Delta m$ & $1.32^{\mathrm{m}}$ & $1.43^{\mathrm{m}}$ & $1.43^{\mathrm{m}}$ \\
\hline$\beta$ & 0.23 & 0.21 & 0.21 \\
\hline Spectrum of A & G8III - IV & G8III - IV & G8III - IV \\
\hline Spectrum of B & & & $\sim \mathrm{F}$ subgiant \\
\hline$M_{V}$ of A & & $0.9^{\mathrm{m}}$ & $0.9^{\mathrm{m}}$ \\
\hline$M_{V}$ of B & & $2.3^{\mathrm{m}}$ & $2.3^{\mathrm{m}}$ \\
\hline
\end{tabular}

\section{8. $\beta$ LMi}

There are spectroscopic, relative (component B w.r.t. A) and speckle-interferometric (in 1975-1995) observations of this pair. However, there are too few observations at periastron. The photocentric semi-major axis and masses of the components were never determined from observations before our research. The orbit by Soderhjelm (1999) and that in the Sixth catalog as well as our orbit (see Table 9) practically coincide in the apparent plane and look as one ellipse with $a_{\mathrm{pm}}=0.13$ in Fig. 8 . The results of the speckle-interferometric observations with the assumption $B-\beta=0.36$ are shown in Fig. 8 by a "cloud" of asterisks at one side of the apparent ellipse. They are also in good agreement with all orbits.

R. F. Griffin (2001) obtained the last periastron passage moment $(1999.33 \pm 0.04)$ from radial-velocity observations. Thus, 38.35 years is the most reliable estimation of the period. We have accepted this value.

The uncertainty of the derived component masses is mainly due to the uncertainty of the photocentric semi-major axis. The mass of the component $\mathrm{A}$ is in good agreement with its spectrum G8III-IV, absolute magnitude $+0.9^{\mathrm{m}}$ and $B-V=+0.9$. The low mass of the brighter component relates perhaps to $[\mathrm{Fe} / \mathrm{H}]=+0.17$ according to Taylor (1999). The spectrum of the component $\mathrm{B}$ can be estimated from its absolute magnitude and the derived mass as about for an F subgiant.

\section{9. $\xi$ Aqr}

The data from the HIP $(B-V=+0.175$, A7V spectrum, parallax of 0.18 and absolute magnitude of the component $\mathrm{A}=+1.0$ ) disagree with one another. A better consistency can be obtained with a larger parallax. We accept $0 . ' 22$ for the parallax and 1.9 solar masses for the component $\mathrm{A}$.

There are a few speckle-interferometric observations, but they are inconsistent with each other (the variation of the
Table 10. Two sets of parameters of $\xi$ Aqr.

\begin{tabular}{|c|c|c|}
\hline & Abt & our \\
\hline$a_{\mathrm{pm}}$ & about $7 \mathrm{AU}$ & $00^{\prime} 11 \pm 0.05$ \\
\hline$P$ & 21.947 & $25.5 \pm 3$ \\
\hline$T$ & 1895.2 & $1970.4 \pm 2.5$ \\
\hline$\Omega$ & & $300^{\circ} \pm 17^{\circ}$ \\
\hline$\omega$ & $222^{\circ} .1$ & $270^{\circ} \pm 36^{\circ}$ \\
\hline$e$ & 0.544 & $0.6 \pm 0.2$ \\
\hline$i$ & & $95^{\circ} \pm 12^{\circ}$ \\
\hline$a_{\mathrm{BA}}$ & & $0 ! 28 \pm 00^{\prime} 03$ \\
\hline parallax & & $0.022 \pm 0 .{ }^{\prime} 004$ \\
\hline$\sum M$ & & $3.1 \pm 0.7$ \\
\hline mass of $\mathrm{A}$ & & 1.9 \\
\hline mass of B & & $1.2 \pm 0.6$ \\
\hline$B$ & & $0.39 \pm 0.2$ \\
\hline$\beta$ & & 0 \\
\hline Spectrum of A & A7V & A7V \\
\hline Spectrum of B & white dwarf & white dwarf \\
\hline$M_{V}$ of $\mathrm{A}$ & & $1.9^{\mathrm{m}}$ \\
\hline$M_{V}$ of B & & $>6^{\mathrm{m}}$ \\
\hline
\end{tabular}

distance is larger than 0.2 for a few years) as well as with Hipparcos and other astrometric results.

The photocentric semi-major axis and the component masses have not been determined from observations.

There are a dozen old spectroscopic observations used for the orbit determination by Abt (1965). The elements for that and our orbit are shown in Table 10. Our orbit is shown in Fig. 9. Abt's $a_{\mathrm{pm}}$ was calculated by us with the assumption of $i \approx 90^{\circ}$ and the component A being the photocentre. The orbit by Abt is far from being precise: for example, the used data allow errors of several years in $P$ and $T$. Our data (specially those of Hipparcos) suggest a longer period of about 25.5 years. Thus, one should not compare our and Abt's orbits directly. However, they do not contradict each other.

Our set of parameters of the pair is presented in Table 10. The unseen component could be a white dwarf. The precision of the $a_{\mathrm{BA}}$ and mass of B shown in Table 10 includes the uncertainties of the photocentric orbit and of the accepted mass of the component A, but without the uncertainty of the accepted parallax. Taking into account also the uncertainty 0. '004 of the parallax, this effect would dominate the resulting uncertainty.

Our results for this pair are less accurate than for others. Nevertheless, we provide the orbital inclination which could not be obtained by other means. It can help to search for the unseen component.

\section{Conclusions}

The proposed astrometric material and method seem to be acceptable to calculate photocentric orbits with periods from 10 to 55 years and semi-major axis of apparent ellipses greater than 0. '08 as well as to predict masses and relative positions of unseen components. New photocentric orbits and component masses are calculated for 4 pairs: $\alpha \mathrm{UMa}, \beta \mathrm{LMi}, \delta$ And and $\xi$ Aqr. 

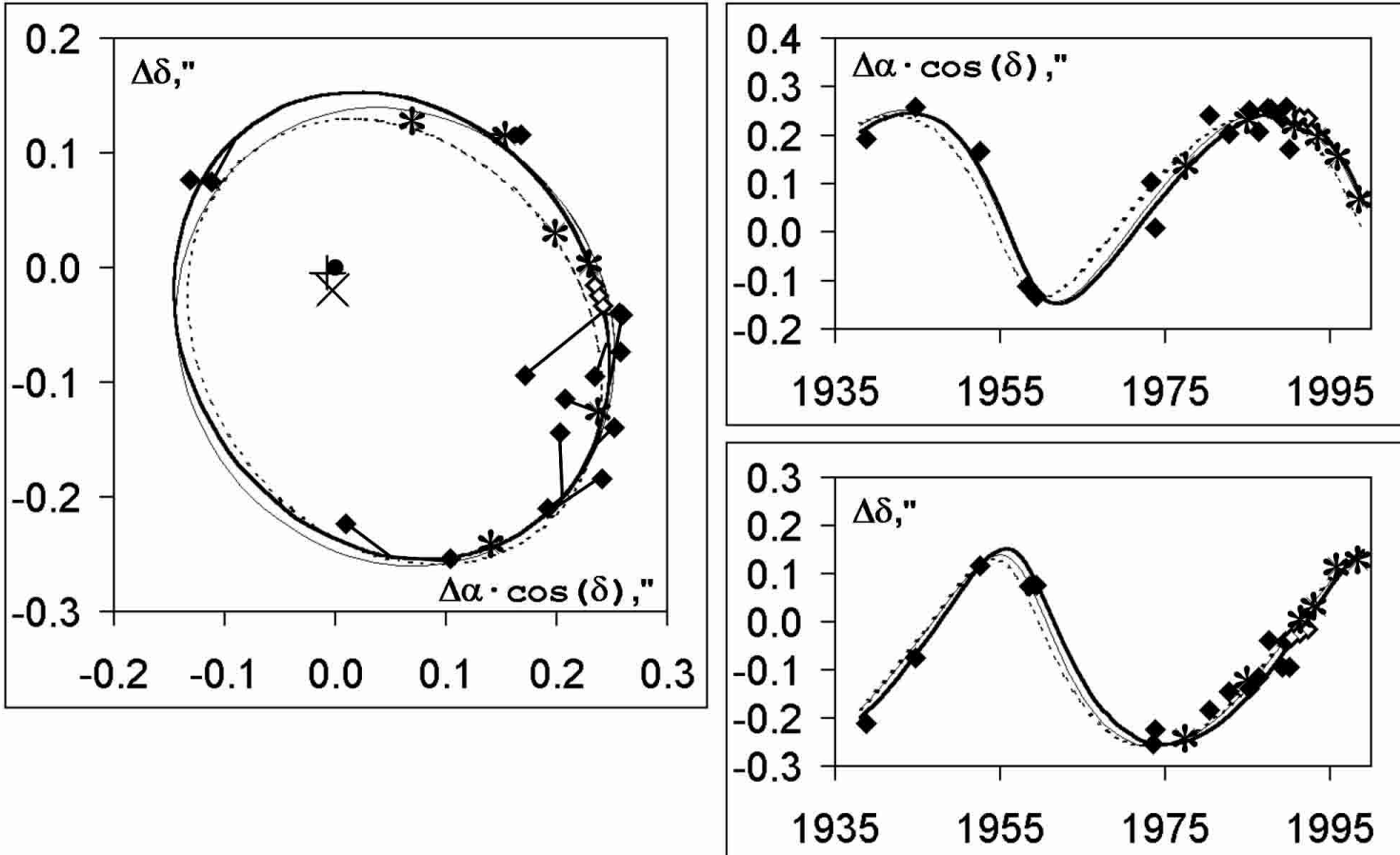

Fig. 7. The photocentric orbits of Dubhe by H-T (dashed curves), Soderhjelm (1999) (thin curves) and our (thick curves) $\left(a_{\mathrm{pm}}=0\right.$ '.21 for all) together with our ground-based (filled diamonds) and Hipparcos (3 open diamonds) results as well as the results of relative observations with the assumption $B-\beta=0.34$ (asterisks).
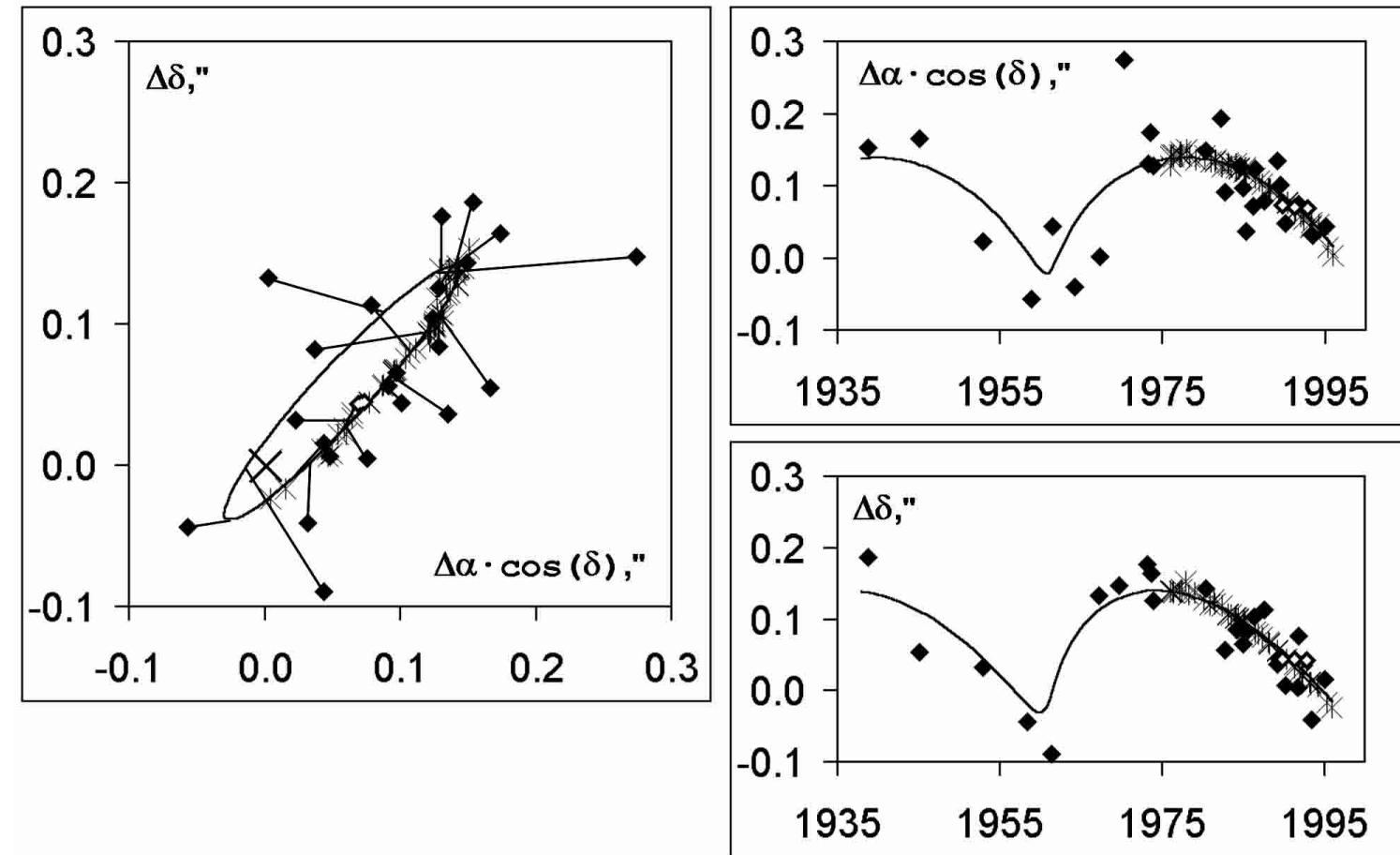

Fig. 8. A photocentric orbit of $\beta$ LMi with $a_{\mathrm{pm}}=0$ '. 13 together with our ground-based (filled diamonds) and Hipparcos (3 open diamonds) results as well as the results of speckle-interferometric observations with the assumption $B-\beta=0.36$ (light asterisks).

Acknowledgements. We thank Dr. R. F. Griffin for providing comments and spectroscopic results on some binaries. We are grateful to Dr. Leslie Morrison for comments to the Carlsberg meridian telescope results. We are thankful to the referee, Dr. H. Schwan, for a lot of reasonable suggestions.
This research has made use of the Centre de Données astronomiques de Strasbourg (CDS), Astronomical Data Center (ADC) at NASA Goddard Space Flight Center and SIMBAD astronomical database.

This research was unaffected by any financially supporting foundation. 


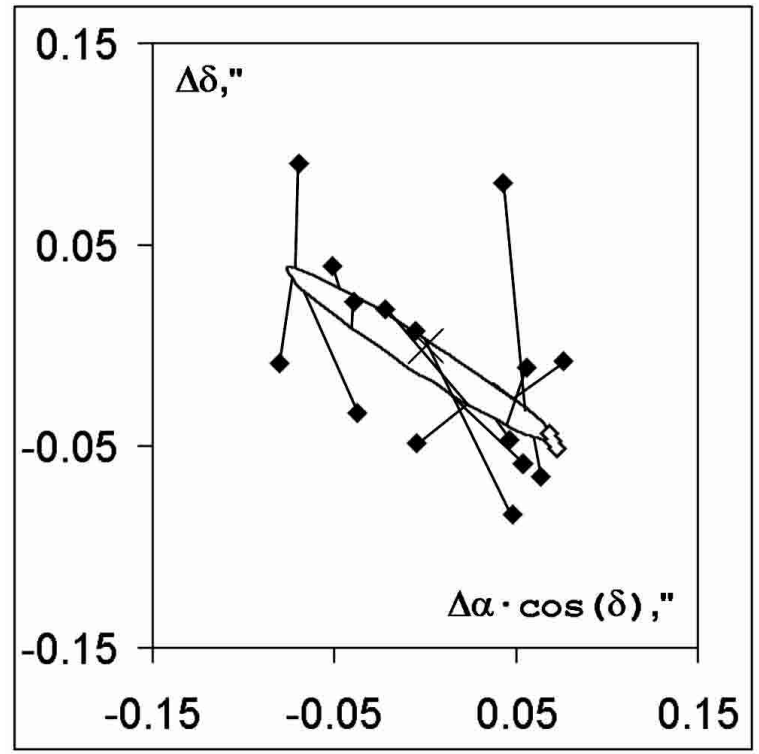

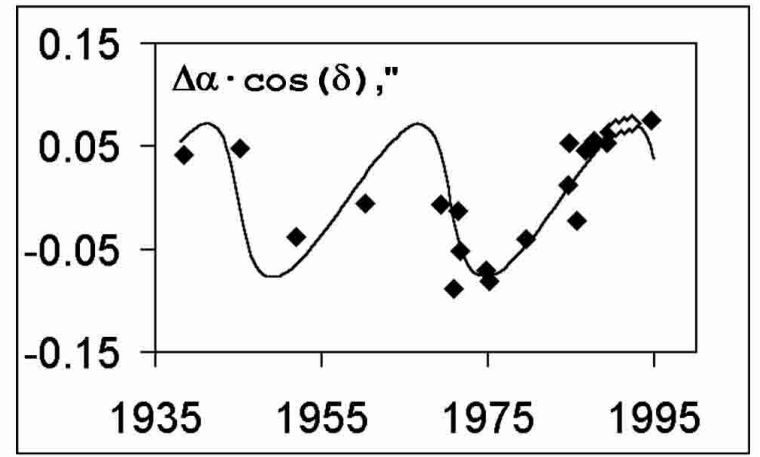

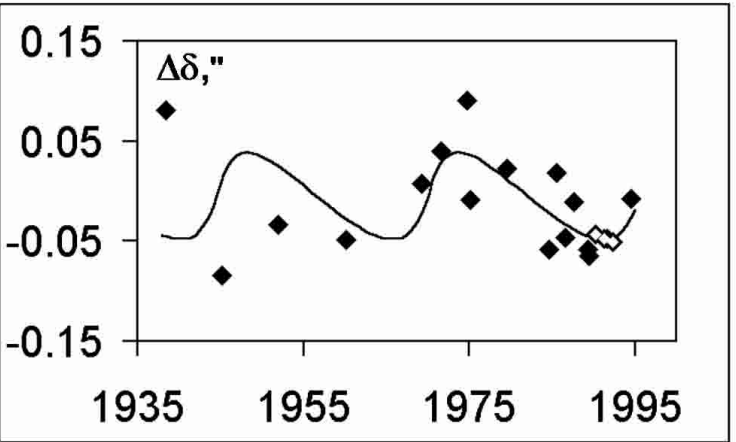

Fig. 9. Our photocentric orbit of $\xi$ Aqr together with ground-based (filled diamonds) and Hipparcos (3 open diamonds) results.

\section{References}

Abt, H. A. 1965, ApJS, 11, 429

Allen, C. W. 1973, Astrophysical quantities (University of London, The Athlone Press)

Augensen, H. J., \& Heintz, W. D. 1992, PASP, 104, 314

Baize, P. 1955, J. Observ., 38, 40

Benest, D., \& Duvent, J. L. 1995, A\&A, 299, 621

Bessel, F. W. 1844, MNRAS, 6, 136

Boccaletti, A., Moutou, C., Mouillet, D., Lagrange, A.-M., \& Augereau, J.-C. 2001, A\&A, 367, 371

Bonnet-Bidaud, J. M., Colas, F., \& Lecacheux, J. 2000, A\&A, 360, 991

Breakiron, L. A., Dabrowski, J. P., \& Gatewood, J. 1975, AJ, 80, 714

Carlsberg Meridian Catalogue La Palma \# 9, 1997, Copenhagen Univ. Obs., Royal Greenwich Obs., Real Inst. y Obs. de la Armada en San Fernando

Drummond, J. D., Christou, J. C., \& Fugate, R. Q. 1995, AJ, 450, 380 ESA, 1997, The Hipparcos and Tycho Catalogues, ESA SP-1200

Fricke, W., Schwan, H., Lederle, T., et al. 1988, Veröff. Astron. Rechen-Institut Heidelberg, 32

Fricke, W., Schwan, H., Corbin, T., et al. 1991, Veröff. Astron. Rechen-Institut Heidelberg, 33

Gatewood, G. D., \& Gatewood, C. V. 1978, ApJ, 225, 191

Girard, T. M., Wu, H., Lee, J. T., et al. 2000, AJ, 119, 2428

Gontcharov, G., \& Kornilov, E. 1997, in Journées 1997, systèmes de référence spatio-temporels, ed. J. Vondrak, \& N. Capitaine (Edition Observ. de Paris and Astron. Inst. Prague), 134

Gontcharov, G. A., Andronova, A. A., \& Titov, O. A. 2000, A\&A, 355,1164

Gontcharov, G. A., Andronova, A. A., Titov, O. A., et al. 2001, A\&A, 365,222

Griffin, R. F. 2001, private communication

Gudehus, D. H. 2001, Amer. Astron. Soc. Meet., Pasadena, CA, June 2001

Hartkopf, W. I., Mason, B. D., \& McAlister, H. A. 1996, AJ, 111, 370
Hartkopf, W. I., \& Mason, B. D. 2001, Sixth Catalog of Orbits of Visual Binary Stars, 2001, URL: http://ad.usno.navy.mil/wds/orb6.html

Hartkopf, W. I., Mason, B. D., Wycoff, G. L., et al. Fourth Catalog of Interferometric Measurements of Binary Stars, 2001, URL: http://ad.usno.navy.mil/wds/int4.html

Heintz, W. D. 1963, Veröff. Sternwarte München, 5, 252

Heintz, W. D. 1984, Publ. Astron. Soc. Pacific, 96, 557

Heintz, W. D. 1997, ApJS, 111, 335

Heintz, W. D., \& Cantor, B. A. 1994, PASP, 106, 363

Irwin, A. W., Fletcher, J. M., Yang, S. I. S., et al. 1992, PASP, 104, 489

Judge, P. G., Jordan, C., \& Rowan-Robinson, M. 1987, MNRAS, 224, 93

Kamper, K. W., Legget, D., \& McCarthy Jr., D. W. 1989, AJ, 98, 686 Kiyaeva, O. V., \& Kalinichenko, O. A. 1998, Publ. Pulkovo observ. (Izvestia Glavnoy...), 213, 233

Kondo, Y., Morgan, T. H., \& Modisette, J. L. 1977, PASP, 89, 163

Lippincott, S. L. 1981, ApJ, 248, 1053

Martin, C., et al. 1998, A\&AS, 133, 149

McCarthy, D., Jr., Hancock, T., Freeman, J., et al. 1993, AJ, 105, 652

Russell, J. L., \& Gatewood, G. D. 1993, PASP, 96, 429

Soderhjelm, S. 1999, A\&A, 341, 121

Strand, K. A. 1951, ApJ, 113, 1

Taylor, B. J. 1999, A\&AS, 134, 523

Tokovinin, A. A. 1997, A\&AS, 124, 75

Turon, C., Crézé, M., Egret, D., et al. 1993, Version 2 of the Hipparcos Input Catalogue, tape version, Bull. Inf. CDS, 43, 5

van den Bos, W. H. 1960, J. Observ., 43, 145

Volet, Ch. 1932, Bull. Astron. Paris, 8, 51

Walker, G. A. H., Walker, A. R., Racine, R., et al. 1994, PASP, 106, 356

Wielen, R. 1997, A\&A, 325, 367

Wielen, R., Schwan, H., Dettbarn, C., et al. 1999, Veröff. Astron. Rechen-Institut Heidelberg, 35 